\title{
Reconstruction of solar UV irradiance in cycle 23
}

\author{
N. A. Krivova ${ }^{1}$, S. K. Solanki ${ }^{1}$, and L. Floyd ${ }^{2}$ \\ 1 Max-Planck-Institut für Sonnensystemforschung, Max-Planck-Str. 2, 37191 Katlenburg-Lindau, Germany \\ e-mail: natalie@mps.mpg.de \\ 2 Interferometrics Inc., 13454 Sunrise Valley Drive, Herndon, VA 20171, USA \\ e-mail: linton.floyd@nrl.navy.mil
}

Received 4 January 2006 / Accepted 26 February 2006

ABSTRACT

\begin{abstract}
Solar irradiance variations show a strong wavelength dependence. Whereas the total solar irradiance varies by about $0.1 \%$ during the course of the solar cycle, variations at the wavelengths around the Ly- $\alpha$ emission line near $121.6 \mathrm{~nm}$ range up to 50-100\%. These variations may have a significant impact on the Earth's climate system. Being almost completely absorbed in the upper atmosphere, solar UV radiation below $300 \mathrm{~nm}$ affects stratospheric chemistry and controls production and destruction of ozone. Models of the solar UV irradiance remain far from perfect, even though considerable progress has been made in modelling the irradiance variations longwards of about 200-300 $\mathrm{nm}$. We show that after correcting for the exposure dependent degradation of the SUSIM channels sampling irradiance at $\lambda>240 \mathrm{~nm}$ (making use of the Mg II core-to-wing ratio) the agreement between model and measurement is significantly improved. At shorter wavelengths the LTE approximation usually made in such models fails, which makes a reconstruction of the solar UV irradiance a rather intricate problem. We choose an alternative approach and use the observed SUSIM UV spectra to extrapolate available models to shorter wavelengths. The model reproduces observed solar cycle variations of the irradiance at wavelengths down to $115 \mathrm{~nm}$ and indicates an important role of UV irradiance variability: up to $60 \%$ of the total irradiance variations over the solar cycle might be produced at wavelengths below $400 \mathrm{~nm}$.
\end{abstract}

Key words. Sun: activity - Sun: faculae, plages - Sun: magnetic fields - solar-terrestrial relations - sunspots - Sun: UV radiation

\section{Introduction}

Solar irradiance variations are reckoned to be prime solar factors exerting influence on the Earth's climate. Of particular interest for climate studies is the variability of the UV part of the solar spectrum (e.g. Haigh 1994, 1996, 1999, 2001; Larkin et al. 2000; Kodera \& Kuroda 2002; Rind 2002; Egorova et al. 2004; Kodera 2004; Matthes et al. 2004; Rozanov et al. 2004).

Regular monitoring of solar UV irradiance started with the Solar Mesosphere Explorer (SME, Rottman 1988), which was operated between 1981 and 1989, but had a rather low accuracy with an uncertainty in UV irradiance of about 10-20\%. With the launch of UARS in 1991 more reliable data became available. Daily measurements at around 120-400 nm were carried out by two instruments, SUSIM (Brueckner et al. 1993) and SOLSTICE (Rottman et al. 1993). They provided valuable information on the absolute level of the solar UV irradiance and the degree of its variation with time. Whereas the Sun's total (integrated over all wavelengths) irradiance changes by about $0.1 \%$ over the course of the solar cycle, its UV irradiance varies by a few percent at 200-300 nm, 10-20\% at 150-200 nm and more than $50 \%$ around the Ly- $\alpha$ emission line near $121.6 \mathrm{~nm}$ (e.g. Floyd et al. 2003a). The amplitude of variations at 300-400 nm remains rather unclear since it is below the $1 \sigma$ uncertainty of both instruments, which is about 2-3\% (Woods et al. 1996).

These irradiance records, while of crucial importance, remain too short to allow definite conclusions about the influence of irradiance variations on climate and need to be extended back in time with the help of models. Most advanced are models of total solar irradiance, which have made great strides toward understanding and reproducing observed irradiance variations on time scales of days to decades (see, e.g., Solanki \& Krivova 2004; Solanki et al. 2005, for overviews). A number of models have also been developed that reconstruct the cyclic component of the irradiance variations on longer time scales, back up to the Maunder minimum (e.g. Lockwood \& Stamper 1999; Solanki \& Fligge 1999; Fligge \& Solanki 2000; Lean 2000). The magnitude of the presumed secular change remains controversial, although some progress has recently been reported (Foster 2004; Wang et al. 2005; Krivova \& Solanki 2005).

Some of these models have also tolerably reproduced variations of spectral irradiance at wavelengths longward of approximately 200 nm (Solanki \& Unruh 1998; Unruh et al. 1999, 2000; Krivova et al. 2003; Fontenla et al. 1999; cf. Fontenla et al. 2004, for an example showing the limits of that model). Also, extended reconstructions of spectral irradiance back to the Maunder minimum exist (Fligge \& Solanki 2000; Lean 2000). Two problems with the modelling of solar UV irradiance are: 1) between 300 and $400 \mathrm{~nm}$ SUSIM data display a far smaller variability than the models (which is generally ascribed to insufficient sensitivity of the measurements); 2) models at shorter wavelengths are often rather simple and less successful. On long time scales, estimates of solar UV irradiance are usually either scaled from total irradiance reconstructions or based on regressions (linear or multiple) with proxy records (e.g. Lean et al. 1995; Lean 2000). On shorter time scales, models are generally more complex. Often Ca II K filtergrams are used to extract information on the evolution of active regions, which is then converted into irradiance (e.g. Lean et al. 1998; Worden et al. 1998, 2001). These models reproduce observations relatively well, indicating that the principal driver of the UV irradiance variations is the surface magnetic activity. 


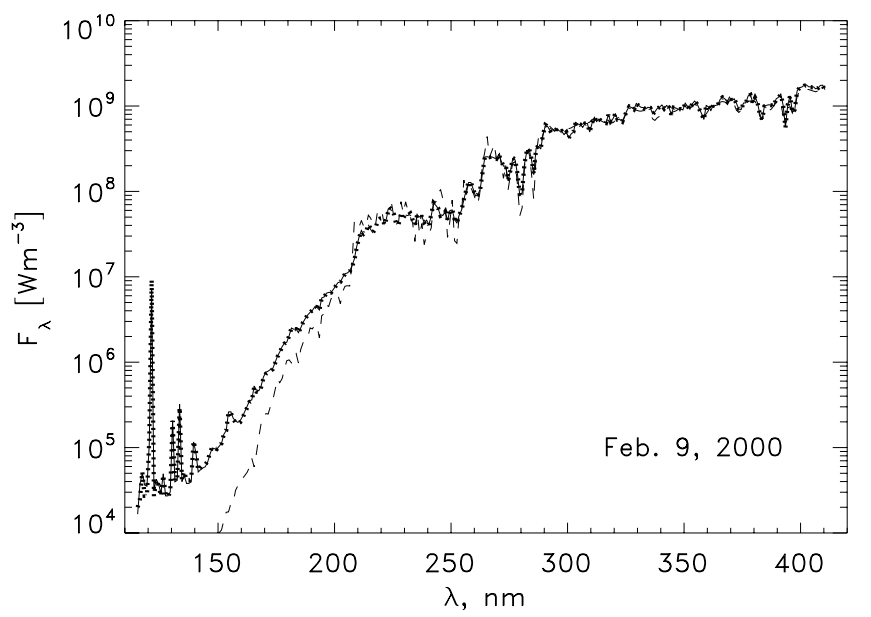

Fig. 1. Solar UV spectrum on February 9, 2000: observed (solid curve) by UARS SUSIM (Brueckner et al. 1993) and modelled (dashed) following Krivova et al. (2003). The dotted line shows the empirically modelled spectrum (see Sects. 2 and 3).

However, an extrapolation of such models to earlier times is not straightforward.

In this paper we address both these problems. By empirically compensating for the exposure-dependent degradation of the sensitivity of SUSIM (making use of the proportionality to the Mg II core-to-wing ratio) we obtain an improved estimate of the solar cycle irradiance variations between 240 and $400 \mathrm{~nm}$. Model atmosphere based reconstructions of solar irradiance variations become more involved in the far UV, because the LTE approximation, which is sufficient to calculate brightness of different photospheric components, fails at these wavelengths (Fontenla et al. 1999; Unruh et al. 1999, see also Fig. 1). Using a non-LTE approximation is one obvious, although tedious, line of attack on the problem (e.g. Fontenla et al. 1999; Haberreiter et al. 2005). In this paper we proceed differently and use observed SUSIM UV spectra to extrapolate available LTE models to shorter wavelengths. This semi-empirical approach can then be used to reconstruct solar UV irradiance at earlier times. Note that we concentrate on wavelengths longer than $115 \mathrm{~nm}$, i.e. where SUSIM data and LTE models are available. The aim of this paper is to demonstrate that this technique gives useful results. The extrapolation to earlier times will be the subject of another paper. For reviews of data and models on yet shorter wavelengths see, e.g., Tobiska et al. (2000); Woods (2002); Woods et al. (2005).

Our approach is presented in Sect. 2, whereas results are described in Sect. 3. We compare modelled and observed variations in the UV irradiance in Sect. 3.1, estimate the contribution of different spectral ranges to the solar cycle irradiance variations in Sect. 3.2 and take a closer look at variations in the range 240-400 nm using signal detection on the SUSIM UARS adjusted signals in Sect. 3.3. Our conclusions are outlined in Sect. 4.

\section{Model}

\subsection{Initial model}

As the starting point we take the model by Krivova et al. (2003). It is based on the assumption that all irradiance variations on time scales of days and longer are solely due to the evolution of the solar surface magnetic features. In order to calculate solar total and spectral irradiance, a 4-component model of the solar photosphere is employed there. It comprises the quiet Sun (or solar surface free of magnetic flux above some threshold), sunspot umbrae, sunspot penumbrae and bright magnetic features including both faculae and the network. Each component is described by the time-independent spectrum calculated (in the LTE approximation) from the corresponding model atmospheres (see Unruh et al. 1999) and the filling factor, which is the fraction of the solar surface covered by the given component at one point in time. A daily sequence of the filling factors is retrieved from the SoHO MDI (Scherrer et al. 1995) continuum images and magnetograms (see Fligge et al. 2000; Krivova et al. 2003, for details). The model reproduces more than $90 \%$ of all total irradiance changes in cycle 23 and has been successfully extended by Wenzler et al. (2004, 2005a,b) using NSO Kitt Peak images to reconstruct solar irradiance in cycles 21 and 22 as well. It also agrees with detrended measurements by the three VIRGO (on SoHO; Fröhlich et al. 1997) spectral channels: blue, green and red centred at 402, 500 and $862 \mathrm{~nm}$, respectively. (Only comparisons with detrended VIRGO spectral channels make sense due to their lack of long-term stability). The model has a single free parameter, $B_{\text {sat }}$, denoting the field strength below which the facular contrast is proportional to the magnetogram signal, while it is independent (saturated) above that. Krivova et al. (2003) obtained a value of $280 \mathrm{G}$ for $B_{\text {sat }}$ from a fit to the VIRGO TSI time series. In the following, we will refer to this model as SATIRE (Solanki et al. 2005; Krivova \& Solanki 2005).

We have calculated solar irradiance spectra for 1497 days between May 1996 and April 2002 with a spectral resolution of $1 \mathrm{~nm}$ in the UV. An example of the reconstructed solar spectrum for February 9, 2000 is shown in Fig. 1 (dashed line) together with the corresponding spectrum recorded by SUSIM (solid). The model and the measurements agree well at wavelengths $\lambda \gtrsim 300 \mathrm{~nm}$ and reasonably at $\lambda \approx 200-300 \mathrm{~nm}$. Shortwards of $\approx 200 \mathrm{~nm}$ the LTE approximation fails increasingly and the model cannot be used directly to estimate solar irradiance. We therefore use spectra recorded by UARS SUSIM to empirically extend our model to shorter wavelengths, as described in Sects. 2.2 and 2.3.

\subsection{SUSIM data}

UARS (Upper Atmosphere Research Satellite) was launched in 1991 carrying, among other experiments, the Solar Ultraviolet Spectral Irradiance Monitor (SUSIM, Brueckner et al. 1993). SUSIM has been monitoring solar irradiance in the range from 115 to $410 \mathrm{~nm}$ with a spectral resolution between 0.15 and $5 \mathrm{~nm}$. Here (except Sect. 3.3) we use the daily level 3BS V21 data with sampling of $1 \mathrm{~nm}$ (Floyd et al. 2003b) obtained from ftp: //susim.nrl . navy.mil. The data are available for the period from October 12, 1991 to April 27, 2002. The wavelength-dependent long-term uncertainty of irradiance measurements $(1 \sigma)$ is around $2-3 \%$ at $\lambda>170 \mathrm{~nm}, \sim 5 \%$ at $\lambda \approx 140-170 \mathrm{~nm}$ and increases to about $10-20 \%$ at $\lambda<140 \mathrm{~nm}$ (Woods et al. 1996; Floyd et al. 1998, 2003b).

In a first step, we identify a wavelength range (henceforward called reference range) over which our model reproduces the observed time variation of the irradiance particularly well and in which the irradiance variations are still above the long-term instrumental uncertainty. As Fig. 1 suggests, the first condition puts a limit of $\lambda \gtrsim 200 \mathrm{~nm}$, whereas the latter one requires that $\lambda \lesssim 300 \mathrm{~nm}$ (e.g. Floyd et al. 2003a). Between 1996 and 2002, a good agreement has been found for the irradiance integrated over, e.g., 220-240 nm (Fig. 2) or 240-260 nm. We choose the 

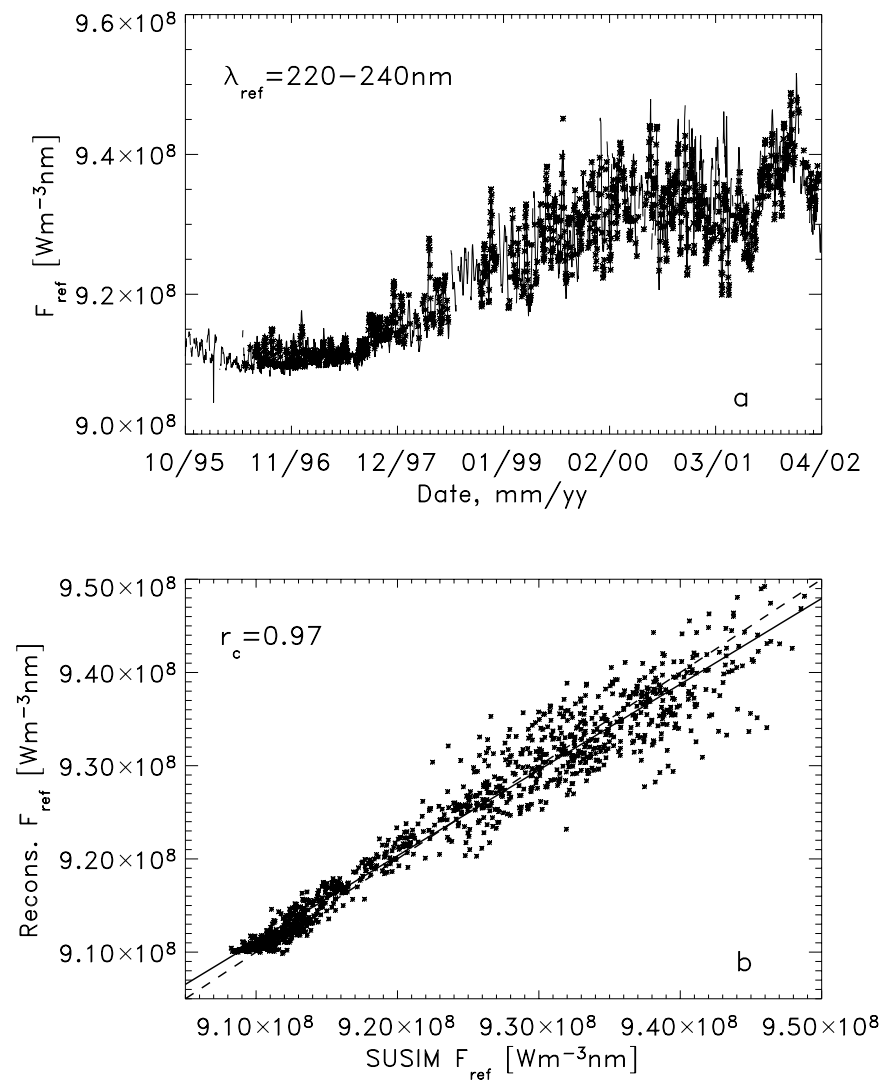

Fig. 2. a) The solar irradiance integrated over the wavelength range 220-240 $\mathrm{nm}$ as a function of time during 1996-2002. The solid line represents SUSIM measurements and asterisks the model by Krivova et al. (2003). b) Reconstructed $F_{\text {ref }}$ vs. SUSIM measurements. The solid line is a regression (correlation coefficient is $r_{\mathrm{c}}=0.97$ ) and the dashed line represents a perfect model fit.

first range as reference since longward of $250 \mathrm{~nm}$ the long-term accuracy of SUSIM becomes comparable to the cyclic variation of the solar irradiance (DeLand et al. 2004).

Our main objective here is to extrapolate the available model from the range where it works well to other wavelengths in the UV using SUSIM data. In order to achieve this, we need to find relations between irradiance, $F_{\lambda}$, at a given wavelength, $\lambda$, and in the reference interval, $F_{\text {ref }}(220-240 \mathrm{~nm})$. For this, we plot for every $\lambda$ the ratio $F_{\lambda} / F_{\text {ref }}$ vs. $F_{\text {ref }}$ on the basis of daily SUSIM data for the whole period 1991-2002.

Two typical examples of such scatter plots at $\lambda=133.5$ and $160.5 \mathrm{~nm}$ are shown in Fig. 3. The presence of two well-defined branches is immediately apparent. The two arms are clearly separated in time, as is apparent from the symbols which refer to different periods: the first 700 days (before November 1993) of SUSIM observations are shown by asterisks, days 701-1800 (between November 1993 and February 1997) by dots and days 1801 onwards by plus signs. Note that the minimum between cycles 22 and 23 occurred at the end of 1996 and it is not immediately clear whether the two branches reflect real differences between the two cycles or a change in the instrument's sensitivity.

Although it is difficult to determine exactly when the behaviour of the relations changes, the time also depends somewhat on the wavelength, the transition period between the two arms does not appear to be symmetrical with respect to the activity minimum. Also the slopes of the two main branches are basically the same, implying a similar behaviour during the two cycles, only with changed absolute values. This is not in favour
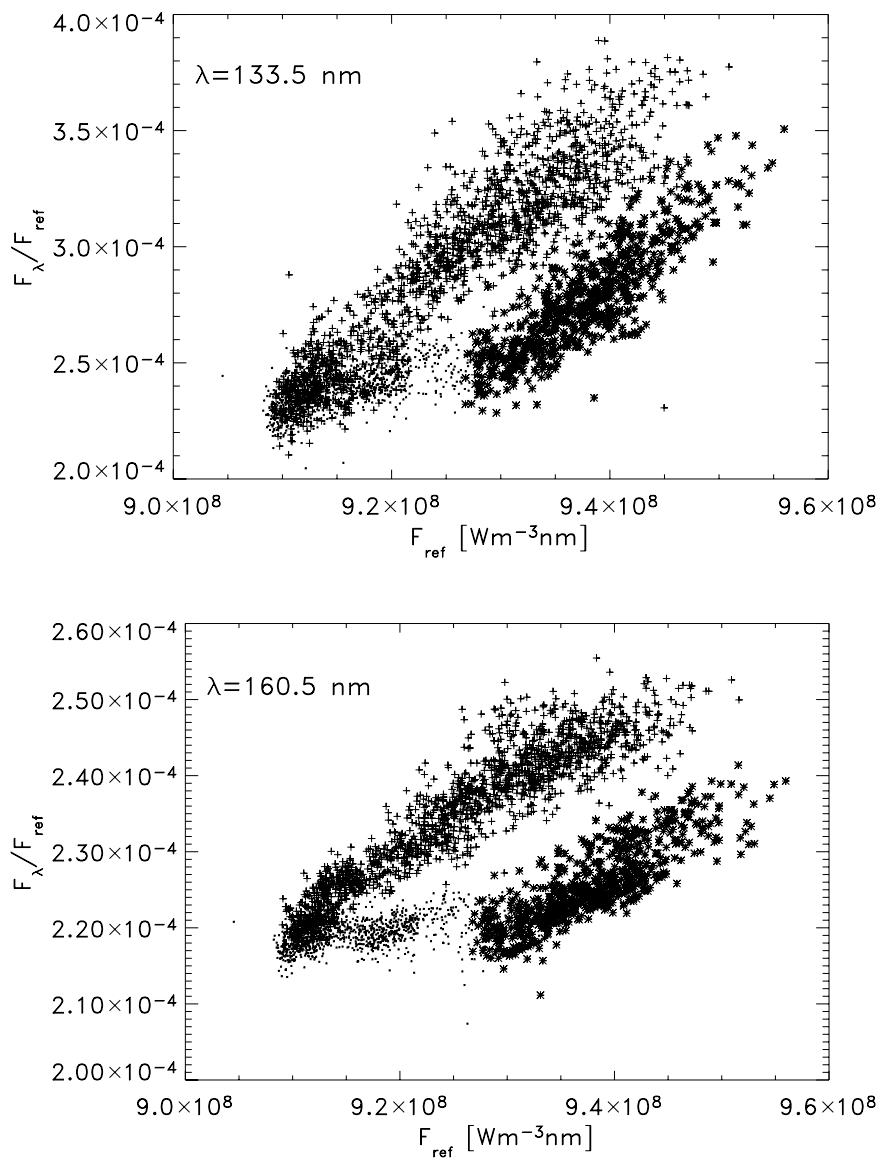

Fig. 3. Ratio $F_{\lambda} / F_{\text {ref }}$ vs. $F_{\text {ref }}$ at $133.5 \mathrm{~nm}$ (top) and $160.5 \mathrm{~nm}$ (bottom) for the whole period of SUSIM observations (1991-2002). Asterisks are used for the first 700 days, dots for days 701-1800 and pluses from the day 1801 onward.

of a real, i.e. solar, change. According to Floyd et al. (2003b) version 21 of the data is not quite free of this kind of problems related to the instrument calibration. The most likely reason is the incorrect estimate of the degradation during the solar minimum period.

In order to check this independently, we have also plotted ratios $F_{\lambda} / \mathrm{Mg}$ II index versus $\mathrm{Mg}$ II index, which is basically the $\mathrm{Mg}$ II core-to-wing ratio. Figure 4a shows the scatter plot for $\lambda=276.5 \mathrm{~nm}$, which is just outside the Mg II absorption feature centred at about $279.9 \mathrm{~nm}$ (see e.g., Viereck \& Puga 1999). The points fall again into two groups, similarly to Fig. 3. Since the $\mathrm{Mg}$ II index is free of degradation effects and the irradiance is not expected to vary significantly differently at these close wavelengths, the figure supports the idea that some degradation effects are still present in this version of SUSIM spectra.

This is further supported by Fig. 4b, showing the correlation coefficient between the two values given in Fig. 4a for 6-month running windows. Dates on the $\mathrm{X}$-axis correspond to the centres of these time intervals. The absolute value of the (negative) correlation is quite high, exceeding 0.8 (dotted line) during most parts of the 1st and 3rd periods shown in Figs. 3 and 4 by asterisks and pluses, respectively (SUSIM days before about 700 and after 1800), but something obviously happened in between. At the end of 1995 and beginning of 1996 the two values become completely uncorrelated. In the first period, before the end of 1993, the correlation is quite high but is gradually decreasing. Therefore the slope of the $F_{\lambda} / F_{\text {ref }}$ vs. $F_{\text {ref }}$ relations might still be affected by the initial degradation from which many radiometric 

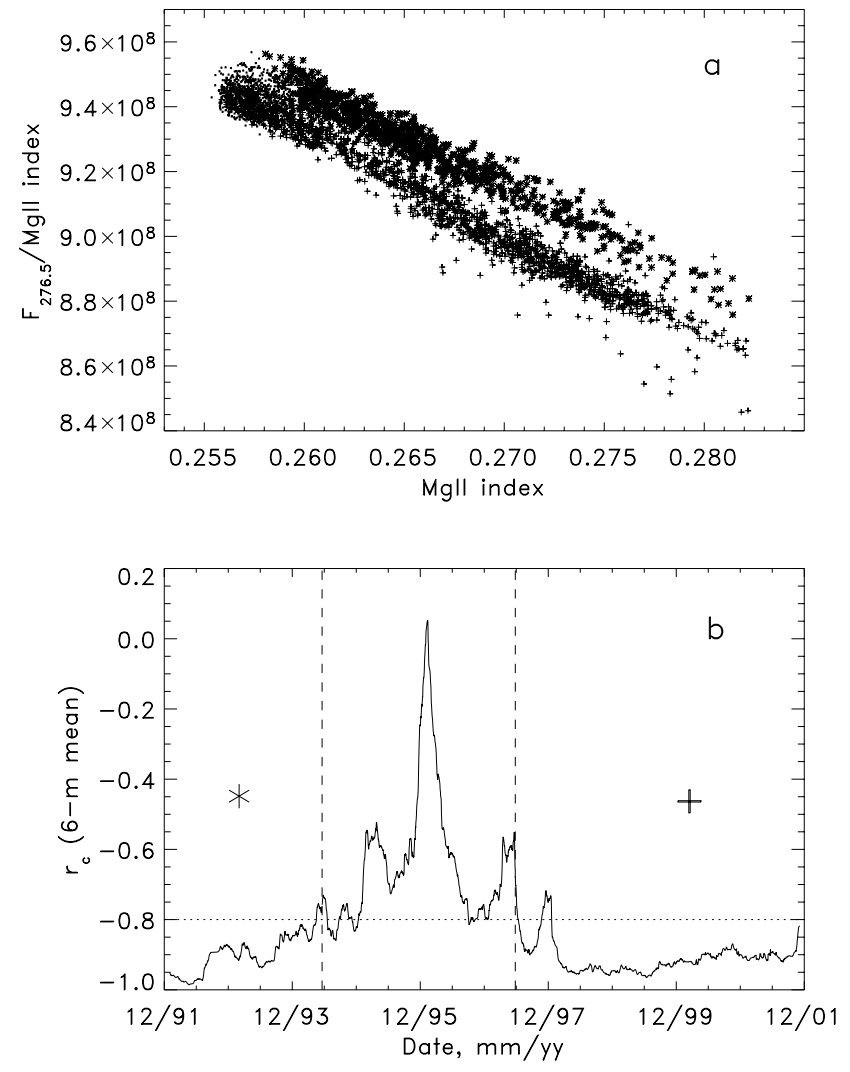

Fig. 4. a) Ratio of the flux at $\lambda=276.5 \mathrm{~nm}$ to the $\mathrm{Mg}$ II index versus $\mathrm{Mg}$ II index. b) The 6-month running mean of the correlation coefficient $r_{\mathrm{c}}$ between the $F_{276.5} / \mathrm{Mg}$ II ratio and $\mathrm{Mg}$ II index. Dates refer to the middle of the corresponding 6-month periods. The horizontal dotted line marks $r_{\mathrm{c}}=-0.8$. Symbols are the same as in Fig. 3 .

instruments suffer. In contrast, the correlation after about 1997 appears relatively stable. Therefore, we use the slope obtained for this period for our further analysis.

For the absolute $F_{\lambda} / F_{\text {ref }}$ value at a given $F_{\text {ref }}$, we need to choose between the two levels. We pick the level corresponding to the initial period of observations (marked by asterisks in Fig. 3) since the absolute value of irradiance probably was more adequately measured at the beginning of the mission. Therefore we correct the ratios for SUSIM days after 1800 to the corresponding levels in the first 700 days. Several examples of the final scatter plots used for the subsequent analysis are shown in Fig. 5. Note that the choice of the SUSIM day 1800 as the starting point is somewhat arbitrary. It is close to the earliest date when the remaining degradation trend does not influence our results. Therefore taking a slightly later date does not affect the final slopes significantly, whereas employing yet earlier data leads to worse correlations.

\subsection{Extension of the initial model}

We now use the corrected SUSIM data to extend our model to shorter wavelengths. We calculate linear regressions to the data $F_{\lambda} / F_{\text {ref }}\left(F_{\text {ref }}\right)$ at every wavelength in the range $115-410 \mathrm{~nm}$. Some examples are shown by straight lines in Fig. 5, with the corresponding correlation coefficients $r_{\mathrm{c}}$ being also indicated. The correlations are typically positive at wavelengths below the reference range (see, e.g., panels $a$ and $b$ of Fig. 5), where irradiance variations are stronger than at $220-240 \mathrm{~nm}$, and positive longwards (panel e), where variations are weaker. At wavelengths in or near the $220-240 \mathrm{~nm}$ range $F_{\lambda} / F_{\text {ref }}$ remains almost constant, so that the correlation is low.

Figure 6, where correlation coefficients for all wavelengths are shown, summarizes this behaviour. The absolute values of $r_{\mathrm{c}}$ are relatively high. Exceptions are a few lines (e.g., $\mathrm{Mg} \mathrm{I}$, $\mathrm{Ca}$ II H \& K, C IV) and the wavelengths in and around the reference range, $220-240 \mathrm{~nm}$. In many such cases, the $F_{\lambda} / F_{\text {ref }}$ ratios are very close to 1 (see Figs. $5 c$ and $5 d$ ), which implies slopes around 0 and leads to low correlations. Of all $r_{\mathrm{c}}$ values, only about $20 \%$ lie between -0.6 and 0.6 (horizontal dotted lines), most of which $(\approx 80 \%$, or $17 \%$ of all points) are within $\lambda \approx 210-260 \mathrm{~nm}$. About $70 \%$ of all points have $\left|r_{\mathrm{c}}\right| \geq 0.8$ (horizontal dashed lines). Note that some prominent far-UV emission lines (e.g., Ly- $\alpha$, O I, C II) are better correlated with $F_{\text {ref }}$ than the continuum radiation at nearby wavelengths. The reason is that the signal is much stronger in these lines than in the nearby continuum below $150 \mathrm{~nm}$. Of particular interest are the $\mathrm{Mg} \mathrm{II} \mathrm{h}$ $\& \mathrm{k}$ lines, which are located at $\lambda>\lambda_{\text {ref }}$, where in general the variability is smaller than at $F_{\text {ref }}$ (negative $r_{\mathrm{c}}$ ). These lines, however, show a larger variability than $F_{\text {ref }}$, so that $r_{\mathrm{c}}>0$.

Finally, if values of $F_{\text {ref }}$ are available (from models or observations), then $F_{\lambda}$ can be calculated using the obtained $F_{\lambda} / F_{\text {ref }}\left(F_{\text {ref }}\right)$ relations at every $\lambda$ between 115 and $410 \mathrm{~nm}$. Below we present a reconstruction of the spectral irradiance between the minimum and maximum of cycle 23 based on the MDI data. This means that the irradiance at $\lambda=220-240 \mathrm{~nm}$ has been reconstructed following Krivova et al. (2003) and then extended to other wavelengths. The method can also be applied to reconstruct solar UV irradiance at earlier times, if $F_{\text {ref }}$ can be modelled by any other means, e.g., back to 1974 based on Kitt Peak magnetograms and continuum images (Wenzler et al. 2005a,b) or even to the end of the Maunder minimum.

\section{Change in the irradiance spectrum between cycle minimum and maximum}

\subsection{Model vs. data}

An example of the solar UV spectrum calculated using the empirical $F_{\lambda} / F_{\text {ref }}\left(F_{\text {ref }}\right)$ relations is presented in Fig. 1 for February 9, 2000. The modelled spectrum (dotted curve) is very similar to the observed one (solid) at all considered wavelengths, improving significantly the initial model (dashed) at wavelengths shorter than about $200 \mathrm{~nm}$. It is also somewhat better in the range between 200 and $300 \mathrm{~nm}$, specially at the locations of strong lines such as $\mathrm{Mg}$ II h+k at $279.6 \mathrm{~nm}$ and $280.3 \mathrm{~nm}$ and the $\mathrm{Mg}$ I line at $285.2 \mathrm{~nm}$. At $\lambda \gtrsim 300 \mathrm{~nm}$ all three spectra are very similar.

The evolution of the irradiance with time at each wavelength is similar to that shown in Fig. 2 for the reference range, except for the amplitude of variations. The amplitude of the solar cycle variations is better represented by Fig. 7. The figure shows the relative irradiance variations between the solar spectrum at activity maximum and minimum. For both the model (dashed line) and the observations (solid), 2-month averages of the spectra were formed at solar activity minimum (October-November 1996) and maximum (April-May 2000). The dates are roughly centred on official sunspot minimum and maximum of cycle 23 (National Geophysical Data Center, Boulder, Colorado: http://www.ngdc.noaa.gov/ stp/SOLAR/solar.html). Note that, as described in Sect. 2.2, we only used SUSIM data starting from February 1997 for the reconstruction. If instead of the official minimum period we use February-May 1997 to represent solar minimum (i.e. within the 

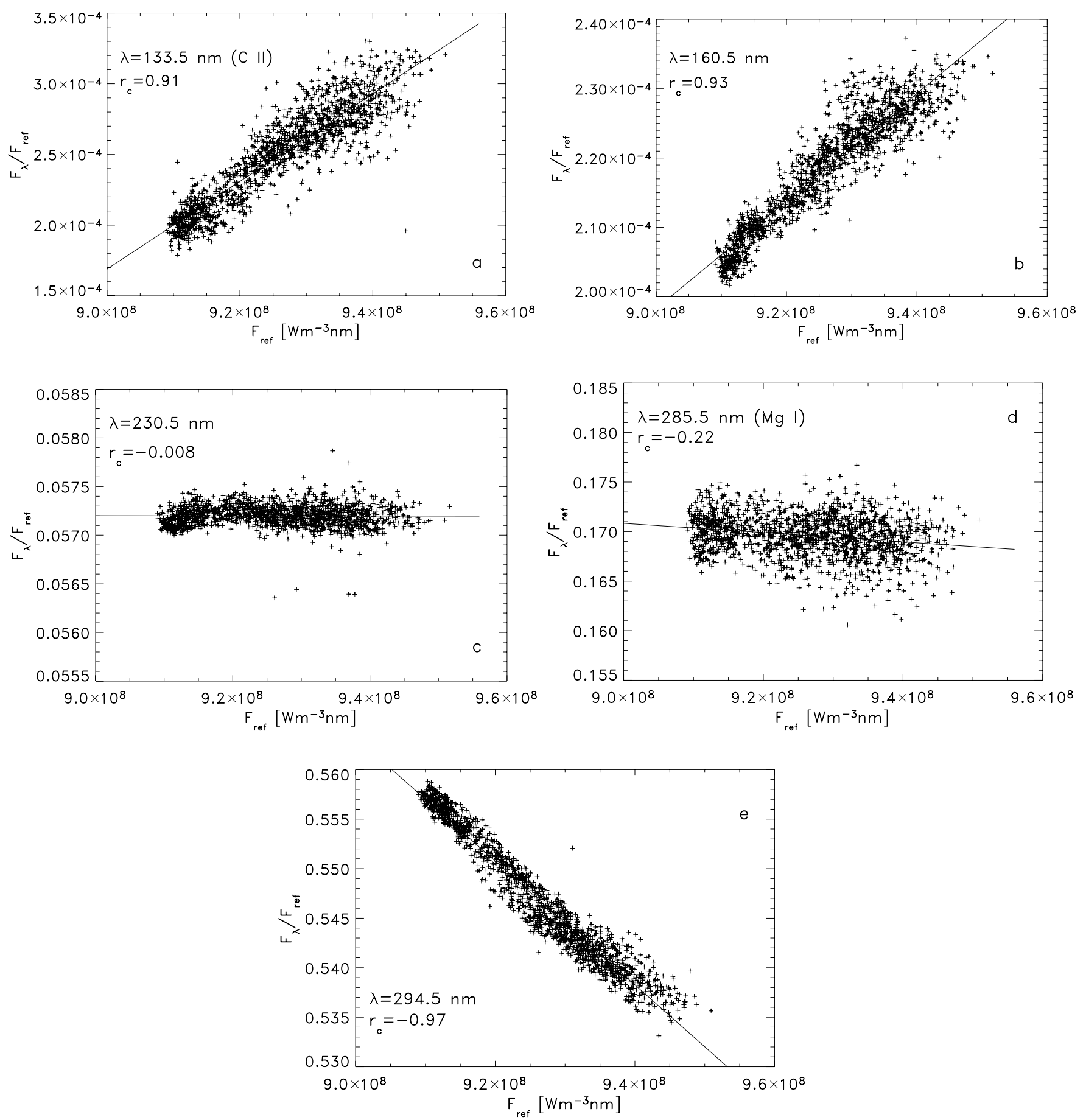

Fig. 5. Ratio $F_{\lambda} / F_{\text {ref }}$ vs. $F_{\text {ref }}$ at $133.5 \mathrm{~nm}$ a), $160.5 \mathrm{~nm} \mathrm{b),} 230.5 \mathrm{~nm}$ c), $285.5 \mathrm{~nm}$ d) and $294.5 \mathrm{~nm}$ e) for SUSIM observations between February 1997 and April 2002. The absolute values of $F_{\lambda} / F_{\text {ref }}$ were corrected to the level of the first 700 days (asterisks in Fig. 3). Plus signs denote SUSIM measurements and the solid lines represent regressions to these data. The corresponding correlation coefficients, $r_{\mathrm{c}}$, are also given.

period of time when SUSIM appeared to be rather stable), the result looks very similar.

Figure 7 shows that the modified model describes the relative change in the solar UV spectrum down to $115 \mathrm{~nm}$ quite well. The solar cycle irradiance variations amount to a few percent at $\lambda=200-300 \mathrm{~nm}, 5-15 \%$ at $\lambda=140-200 \mathrm{~nm}$, increasing to several tens of percent at shorter wavelengths, including Ly- $\alpha$ and may run as high as $\sim 100 \%$ at $\lambda \lesssim 120 \mathrm{~nm}$, in agreement with Floyd et al. (2003a). This figure only shows how well the use of linear regressions between different wavelength regions can be used to represent the evolution of the whole spectrum. The figure basically demonstrates the consistency between the empirical model and the SUSIM measurements. In the wavelength range $<300 \mathrm{~nm}$ the model contains no information beyond what is already contained in the SUSIM data.

The combination of the original model with the empirical extension into the UV covers a broad wavelength range (Fig. 8). With this model we can calculate the contribution of different wavelength ranges to solar irradiance and its variations. Since the combined model covers a broader wavelength range $(115-160000 \mathrm{~nm})$ than previous models or data sets this estimate should constitute an improvement. 


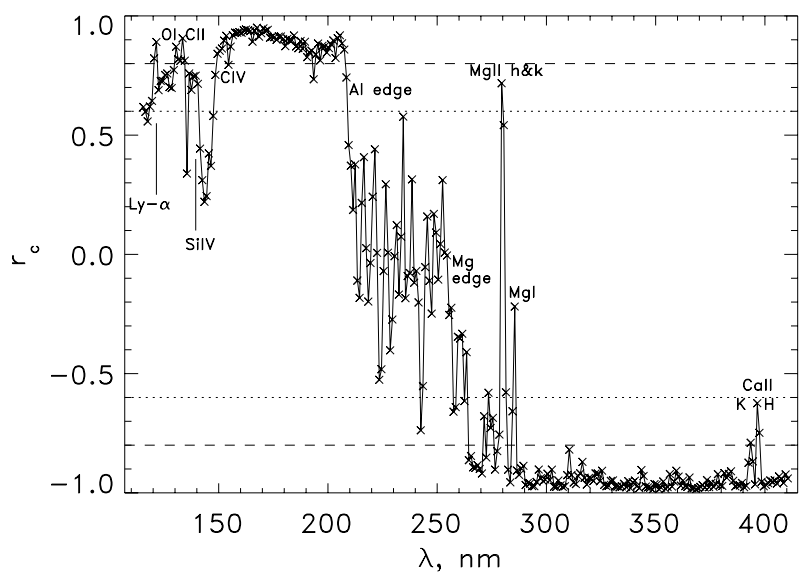

Fig. 6. Correlation coefficient $r_{\mathrm{c}}$ between $F_{\lambda} / F_{\text {ref }}$ and $F_{\text {ref }}$ as a function of wavelength.

\subsection{Spectral distribution of irradiance variations}

The long term uncertainty of SUSIM measurements becomes comparable or higher than the solar cycle variations at around 250 and $300 \mathrm{~nm}$, respectively. A resulting artifact is that the relative difference between solar irradiance at activity maximum and minimum as estimated from SUSIM data (and thus in the modified model) is sometimes negative (see Fig. 7b) at $\lambda \gtrsim 280 \mathrm{~nm}$. On the other hand, the initial model describes solar spectrum in this range quite well (see Fig. 1), does not show this artifact and always gives a positive change in irradiance from activity minimum to maximum. Therefore we use the initial model at $\lambda>280 \mathrm{~nm}$ and the empirically modified one at shorter wavelengths. The exact choice of the wavelength at which the two models are merged is of no importance.

The calculated contribution of different wavelength ranges to solar irradiance and its 11 -year cycle variation is presented in Fig. 9. The length of one $\lambda$-step is $\approx 40 \mathrm{~nm}$ below $200 \mathrm{~nm}, 50 \mathrm{~nm}$ between 200 and $400 \mathrm{~nm}, 100 \mathrm{~nm}$ between 400 and $1000 \mathrm{~nm}$. The longer wavelengths are combined into larger bins of $500 \mathrm{~nm}$ each since they are not of prime interest here. Although almost $1 / 3$ of solar energy comes from these long wavelengths $(>1000 \mathrm{~nm})$, their contribution to the irradiance variations is negligible (it is negative at $1000-3000 \mathrm{~nm}$ due to sunspots and is about $1-1.5 \%$ at yet longer $\lambda$ ). The figure shows the high role of the UV irradiance in the TSI change: about a quarter of all changes comes from 350-400 nm, and the wavelength interval 300-400 nm accounts for about $40 \%$ of the TSI variation. Another $20 \%$ are due to irradiance at $200-300 \mathrm{~nm}$. Only about a quarter of the TSI variations are produced longwards of $500 \mathrm{~nm}$.

The contribution of the Sun's UV irradiance to the TSI was estimated using different observational data by Lean (1989) and Mitchell \& Livingston (1991) for solar cycle 21, as well as by Lean et al. (1997) for cycle 22. Lean (1989) employed solar UV data obtained by SBUV (the Solar Backscatter Ultraviolet experiment on Nimbus-7) and the solar spectrometer on SME (the Solar Mesosphere Explorer) in combination with TSI measurements by ACRIM (the Active Cavity Radiometer) on SMM to estimate the contribution of the solar UV irradiance between 200 and $400 \mathrm{~nm}$ to the total irradiance for cycle 21 (between 1981 and 1985). She found that the decrease in the UV irradiance at 200-300 nm might account for about $19 \%$ of the TSI decrease. For $300-400 \mathrm{~nm}$, the estimated contribution was about $13 \%$ but was rather uncertain due to the long-term uncertainty similar to that displayed by the SUSIM data. Lean et al. (1997) used
Nimbus 7 ERB and SOLSTICE measurements in combination with the sunspot darkening and facular brightening (from $\mathrm{Mg}$ II proxy) indices to parameterise variations of the solar irradiance between 200 and $400 \mathrm{~nm}$. They estimated variations over the cycle 22 (between 1989 and 1986) at about $13 \%$ and $17 \%$ in the ranges $200-300 \mathrm{~nm}$ and $300-400 \mathrm{~nm}$, respectively. Mitchell \& Livingston (1991) used solar irradiance spectra obtained at maximum and minimum of cycle 21 at Kitt Peak and compared the changes in the 300-560 $\mathrm{nm}$ wavelength range with those in ACRIM TSI data. The main uncertainty in their model comes from the unknown facular contrast. Note that the facular contrast given by our model atmospheres (Unruh et al. 1999) is closer to the higher value employed by Mitchell \& Livingston (1991). Their estimates (depending on the assumption regarding the facular contrast) are listed in Table 1 together with those by Lean (1989), Lean et al. (1997) and the results of our modelling. Values obtained from an earlier version of SATIRE (Unruh et al. 1999) are also given. In order to model solar cycle variations in the irradiance, they employed typical values of the filling factors for sunspots and faculae rather than real ones for given dates. Our results are thus in good agreement with the observational estimates for cycle 21 of Lean (1989) at 200-300 nm and Mitchell \& Livingston (1991) at 300-500 nm. Some difference with the results by Unruh et al. (1999) is due to the uncertainties at shorter wavelengths $(\$ 200 \mathrm{~nm})$ in the earlier model. Part of difference between all estimates may also be that they usually do not consider the same maximum and minimum periods (e.g., different cycles).

Since the radiative transfer model gives somewhat higher $\Delta F$ values than SUSIM measurements between 300 and $400 \mathrm{~nm}$ it is not surprising that the fraction comes out larger than found by, e.g., Lean (1989) and Lean et al. (1997). If we use the original SUSIM data below $410 \mathrm{~nm}$ and combine them with the SATIRE model at longer wavelengths then we obtain the values in the 4th column of Table 1 . They agree with the estimates by Lean (1989) and Lean et al. (1997) in the range between 300 and $400 \mathrm{~nm}$ and those by Lean (1989) at 200-300 nm but differ from the results of Mitchell \& Livingston (1991) at the wavelengths between 300 and $560 \mathrm{~nm}$. We note, however, the large uncertainty in the SUSIM data at these wavelengths, which have a systematic component due to degradation, as we discuss in the next section.

\subsection{Solar cycle variation for $240-400 \mathrm{~nm}$ using signal detection on the SUSIM UARS adjusted signals}

Over the nearly 14 year lifetime of the SUSIM experiment, there were four distinct working channels each of which were scanned daily. The three changes in the working channel optical element set were needed because of its progressive loss of responsivity. This responsivity degradation has been found to be approximately proportional to UV exposure. The degradation mechanism is not completely known, but is understood to be caused by UV polymerization of hydrocarbon contaminants on optical surfaces (Floyd et al. 1998; Floyd 1999). The growing polymerized layer becomes increasingly opaque to incoming UV radiation.

If unaccounted for, this degradation could mask the true solar variation that is incorporated in the SUSIM measurements. Calibration of the changing responsivity of SUSIM's working channel was provided through a combination of measurements of four onboard deuterium calibration lamps and solar measurements by less frequently exposed reference optical channels (Prinz et al. 1996; Floyd et al. 1996). The relative accuracy of this method is understood to be roughly $1-2 \%$ over the 

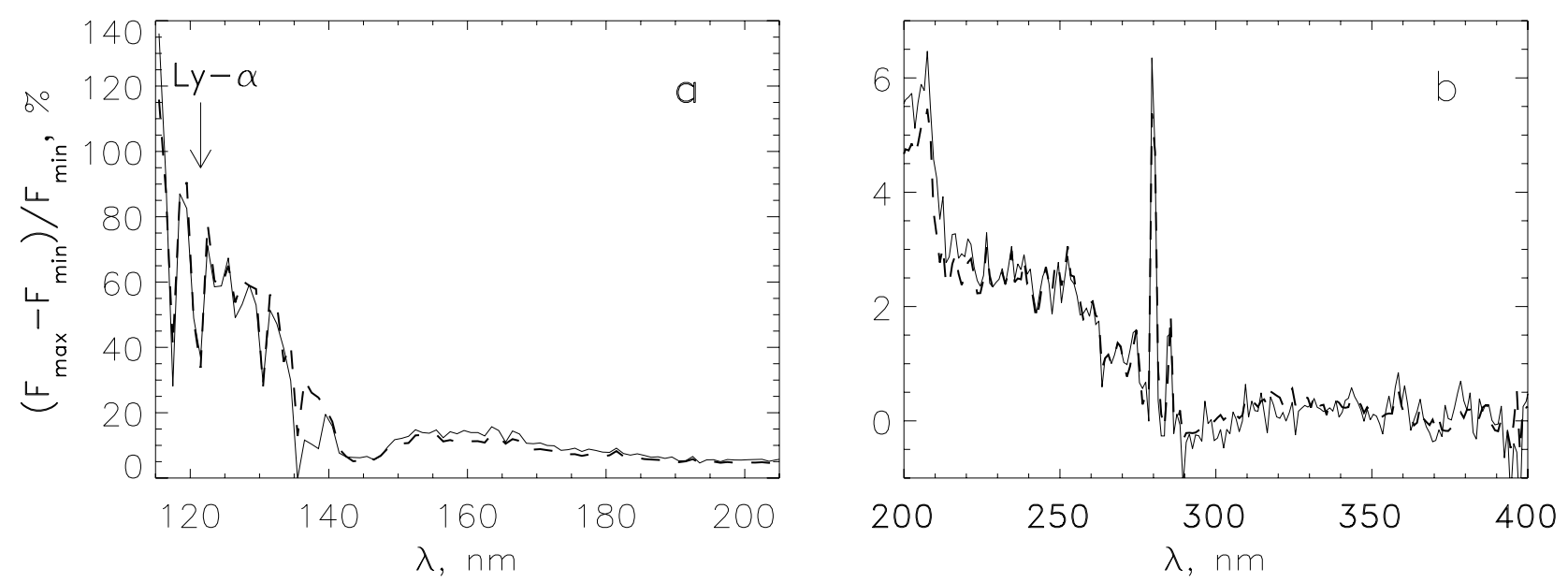

Fig. 7. Relative irradiance variations between the solar spectrum at activity maximum (2-month average, April-May 2000) and minimum (2-month average, October-November 1996) at 115-210 $\mathrm{nm}$ a) and 200-420 $\mathrm{nm}$ b). The solid line represents SUSIM measurements and the dashed line our reconstruction.

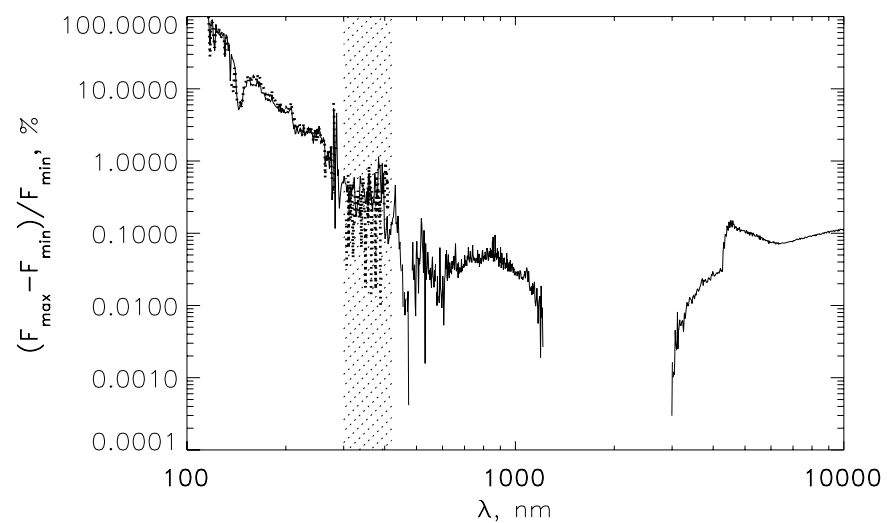

Fig. 8. The combined model (solid line, see text) and SUSIM data (dotted). The hatched area marks the region where the uncertainty of SUSIM data exceeds solar cycle variations.

long-term (i.e. years) time scales. Because this is a very significant portion of the solar cycle variation for wavelengths above about $263 \mathrm{~nm}$ and is actually larger than the solar cycle variation for wavelengths above about $290 \mathrm{~nm}$, the lamp and redundant channel calibration method does not provide a sufficiently accurate gauge of the solar cycle variation for these wavelengths.

Here we present a different approach to establishing the solar variation through the detection of the presence and magnitude of the solar activity signal in the daily measurements of the SUSIM working channel. Solar UV spectral irradiance time series have been found to be linearly related to the time series of the $\mathrm{Mg}$ II core-to-wing ratio index by a number of experiments and wavelength ranges (Heath \& Schlesinger 1986; DeLand \& Cebula 1993, 1998; Viereck et al. 2001, 2004; Floyd et al. 2005). Accordingly, through correlative comparisons with the Mg II index, we attempt to infer the variation from the solar cycle signal embedded in the solar irradiances.

Floyd (1999) found empirically that decline in the responsivity of the working channel follows the form:

$R(x)=c_{0}+c_{1} \log \left(1+x / c_{2}\right)$

where $R$ is the wavelength-dependent responsivity, $x$ is the timedependent UV exposure, and the $c_{n}$ are the determined constants. Assuming a linear dependence of irradiance in the simplified (but still wavelength-dependent) form of the instrument equation

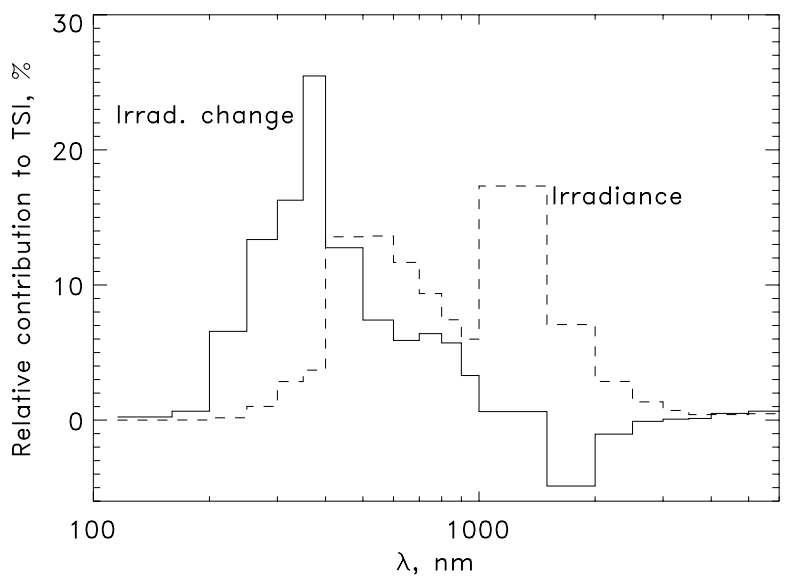

Fig. 9. The relative contribution of different wavelength ranges to the TSI (dashed histogram) and its solar cycle variations (solid histogram). Note different size of bins: about $40 \mathrm{~nm}$ below $200 \mathrm{~nm}, 50 \mathrm{~nm}$ between $200 \mathrm{~nm}$ and $400 \mathrm{~nm}, 100 \mathrm{~nm}$ between 400 and $1000 \mathrm{~nm}$ and $500 \mathrm{~nm}$ at yet longer wavelengths.

$S=I R$, where $S$ is the measured signal and $I$ is the irradiance, we find

$S(t)=I R=\left(1+a_{3} M\right)\left(a_{0}+a_{1} \log \left(1+x / a_{2}\right)\right)$,

where $t$ is time, $M$ is the $\mathrm{Mg} \mathrm{II}$ index and $a_{n}$ are constants to be determined. Note that although the $1+a_{3} M$ factor is not equal to the irradiance, it is proportional to it. The determined constant $a_{3}$ determines the fractional solar cycle variation $f$ of the solar minimum level as:

$f=\frac{a_{3}\left(\bar{M}\left(t_{\max }\right)-\bar{M}\left(t_{\min }\right)\right)}{1+a_{3} \bar{M}\left(t_{\min }\right)}$

where $\bar{M}$ is the $\mathrm{Mg}$ II index smoothed using an 81-day running mean and $t_{\max }$ and $t_{\min }$ are the days of maximum and minimum $\bar{M}$. We use the running mean to distinguish the solar cycle variation from that of solar rotation, the other large periodicity present in these data. Under the assumption that the solar UV irradiance varies strictly in proportion to the $\mathrm{Mg}$ II index, a determination of $a_{3}$ allows the estimation of the solar cycle variation for any period for which the Mg II index is available.

The solar UV measurements used in this preliminary study are those of the SUSIM long wavelength optical channel 
Table 1. The relative irradiance variation between activity maximum and minimum in different wavelength ranges. Column (1) lists the wavelength intervals, Col. (2) the contribution of the irradiance in these intervals, $F_{\lambda}$, to the total solar irradiance, $F_{\text {tot }}$, Cols. $(3-8)$ the corresponding contributions of the relative irradiance change, $\Delta F_{\lambda}$, to the relative change in the TSI, $\Delta F_{\text {tot }}$ estimated: Cols. $(3,4)$ in this work using Col. (3) the extrapolated and original SATIRE models below and above $280 \mathrm{~nm}$, respectively, and Col. (4) SUSIM V21 data and the SATIRE model below and above $410 \mathrm{~nm}$, respectively; Col. (5) by Lean (1989) and Col. (6) Lean et al. (1997), Col. (7) by Mitchell \& Livingston (1991), Col. (8) by Unruh et al. (1999). The two numbers in the case of Mitchell \& Livingston (1991) are for two different assumptions regarding the facular covering factor, $f$, and facular contrast at disc centre, $C_{\mathrm{F}}$.

\begin{tabular}{|c|c|c|c|c|c|c|c|}
\hline \multirow[t]{3}{*}{$\lambda \lambda$ range, $\mathrm{nm}$} & \multirow[t]{3}{*}{ 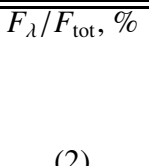 } & \multicolumn{6}{|c|}{$\bar{\Delta} \Delta F_{\lambda} / \Delta F_{\text {tot }}, \%$} \\
\hline & & model & $\begin{array}{l}\text { SUSIM V21 } \\
(1<410 \mathrm{~nm})\end{array}$ & L-89 & Lea-97 & ML-91 & USF-99 \\
\hline & & cycle 23 & 23 & 21 & 22 & 21 & - \\
\hline $115-200$ & 0.009 & 0.9 & 1.3 & - & - & - & - \\
\hline $200-300$ & 1.2 & 20.0 & 19.0 & 19.1 & 12.9 & - & - \\
\hline $300-400$ & 6.5 & 41.8 & 15.1 & 13.0 & 17.9 & $32.7^{a} / 39.9^{b}$ & 31.5 \\
\hline $400-500$ & 13.5 & 12.8 & 31.8 & - & - & $3.2^{a} / 15.4^{b}$ & 17.3 \\
\hline $500-600$ & 13.6 & 7.4 & 9.8 & - & - & $-4.1^{a, c} / 2.9^{b, c}$ & $7.7^{c}$ \\
\hline $600-700$ & 11.6 & 5.9 & 7.8 & - & - & - & - \\
\hline $700-800$ & 9.3 & 6.4 & 8.5 & - & - & - & - \\
\hline $800-900$ & 7.4 & 5.7 & 7.6 & - & - & - & - \\
\hline $900-1000$ & 6.0 & 3.3 & 4.4 & - & - & - & - \\
\hline 1000-3000 & 28.8 & -5.5 & -7.2 & - & - & - & - \\
\hline $3000-160000$ & 2.0 & 1.4 & 1.8 & - & - & - & - \\
\hline
\end{tabular}

L-89: Lean (1989). Lea-97: Lean et al. (1997). ML-91: Mitchell \& Livingston (1991). USF-99: Unruh et al. (1999).

${ }^{a} f=0.031 \pm 0.005, C_{\mathrm{F}}=1.00 ;^{b} f=0.033 \pm 0.005, C_{\mathrm{F}}=1.02{ }^{c}$ for $\lambda=500-560 \mathrm{~nm}$.

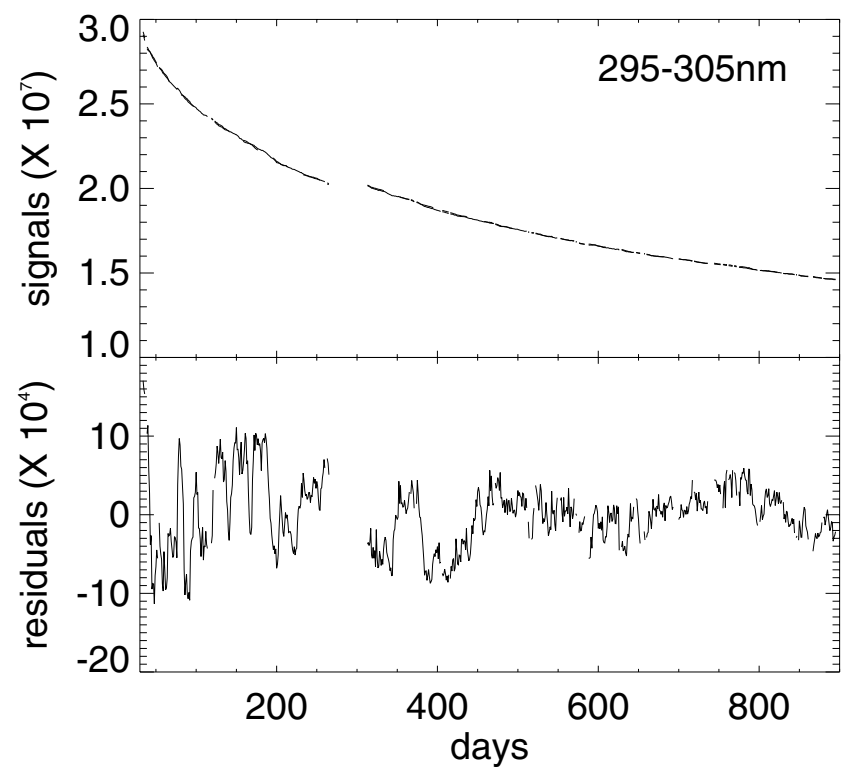

Fig. 10. The solid curve of upper panel represents the integrated SUSIM signals for the 295-305 $\mathrm{nm}$ wavelength range plotted as a function of exposure given in arbitrary units. The dashed line represents the least squares fit of Eq. (2). The corresponding residuals are displayed in the bottom panel.

(237-412 nm) at $1.1 \mathrm{~nm}$ resolution for the period of time from the start of the SUSIM experiment (11 October 1991) through the first major change in the working channel (22 February 1994) during the declining phase of solar cycle 22. Floyd (1999) gives additional details of the analysis method; a more complete study making use of other time periods and optical channels is in preparation (L. Floyd, L. Herring, \& J. Cook, in preparation). Corrections for all instrumental effects except for responsivity were applied to the raw spectral irradiance measurements. Next, these processed measurements were binned over wavelength ranges having widths of 5-20 nm. These specific

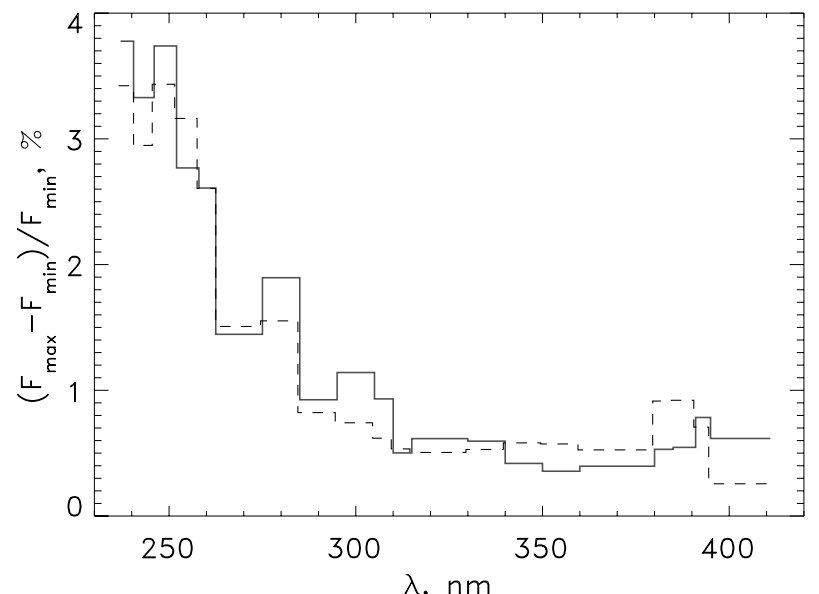

Fig. 11. The percentage variation in the solar UV spectral irradiances above $240 \mathrm{~nm}$ for solar cycle 23 from the SATIRE-based model (dashed line; SATIRE above $280 \mathrm{~nm}$ and its empirical extension based on SUSIM level 3BS V21 data at shorter wavelengths) and from the Mg II index model applied to the SUSIM uncalibrated irradiance signals measured during 1991-1994 (solid).

bin selections were required to prevent corruption of the results by known instrumental effects. The integrated signals for each bin were fit by the function given above in Eq. (2) using the NOAA SEC composite Mg II index (Viereck et al. 2004), and the $a_{n}$ determined. Figure 10 displays the integrated signals for 295-305 nm, the fitted function, and the corresponding residuals. From the values of $a_{3}$, the variation for each wavelength region between the minimum and maximum of solar cycle 23 was estimated.

This variation is displayed in Fig. 11 along with corresponding estimates obtained for the same bins and same periods from the SATIRE-based model (i.e. original SATIRE model above $280 \mathrm{~nm}$ and its SUSIM-based (V21) empirical extrapolation as 
described in Sect. 3.2 for wavelengths below $280 \mathrm{~nm}$ ). Note that the results of the $\mathrm{Mg}$ II index model above $395 \mathrm{~nm}$ are relatively uncertain due to stronger unresolved instrumental effects (e.g. stray light) at those wavelengths. On the other hand, the variation in the chromospheric Ca II H and K (393 and 397 nm, respectively) lines might be not accurately reproduced by the SATIRE atmosphere models. Otherwise, the two estimates agree very well with each other and imply the contribution of the UV part (below $400 \mathrm{~nm}$ ) of the solar spectrum to the total solar cycle irradiance variations of about $60 \%$.

Lean et al. (1997) have previously estimated the solar cycle variation in the UV largely from three years of SOLSTICE data over a similar time period 1991-1994. The approach to the detection of the long-term UV variation in their data sets differs from that given here in two major respects. First, Lean et al. (1997) compare the irradiances with separate proxies for the sunspot darkening and facular brightening whereas the estimation method presented here utilizes only the $\mathrm{Mg}$ II index facular proxy. Second, to establish the long term variation in that study, signal detection is performed on detrended versions of both the solar proxies (i.e., those representing sunspot darkening and facular brightening) and UV irradiances. Such an analysis depends on the assumption that the correspondence between the variations during solar rotation and those on longer (e.g. solar cycle) time scales is the same for the UV irradiances and proxies. While this appears to be usually true for UV wavelengths below about 263 nm (e.g., DeLand \& Cebula 1998; Floyd et al. $2003 \mathrm{~b}$ ), the level of correspondence has not been established for longer wavelengths. By contrast, because no detrending of data or proxy is used in the present study, these results do not depend on this assumption. The lack of a sunspot blocking proxy in the present study may cause errors in the given estimates. However, for time scales longer than solar rotation, the long-term effects of sunspot blocking and facular brightening both generally follow the level of solar activity. Accordingly, because we consider only the long-term variation, such errors may well be minimal.

\section{Conclusions}

The current results based on two independent estimates greatly increase the fraction of solar cycle total irradiance variations due to UV wavelengths: up to about $60 \%$ of the total irradiance variations are produced at wavelengths below $400 \mathrm{~nm}$. If correct, this would significantly enhance the importance of UV irradiance variability and provide support for investigating the response of climate to variations in short wavelength radiation.

The current investigation also highlights the pressing need for higher accuracy measurements of the solar irradiance between 300 and $400 \mathrm{~nm}$.

Acknowledgements. We thank Y. C. Unruh for helpful discussions. This work was supported by the Deutsche Forschungsgemeinschaft, DFG project number SO 711/1-1. The analysis of the SUSIM data was supported by the NASA Living With a Star Targeted Research and Technology program, LWS04-0093-0149.

\section{References}

Brueckner, G. E., Edlow, K. L., Floyd, L. E., Lean, J. L., \& Vanhoosier, M. E. 1993, JGR, 98, 10695

DeLand, M. T., \& Cebula, R. P. 1993, JGR, 98, 12809

DeLand, M. T., \& Cebula, R. P. 1998, JGR, 103, 16251
DeLand, M. T., Floyd, L. E., Rottman, G. J., \& Pap, J. M. 2004, Adv. Sp. Res., 34,243

Egorova, T., Rozanov, E., Manzini, E., et al. 2004, GRL, 31, 6119

Fligge, M., \& Solanki, S. K. 2000, GRL, 27, 2157

Fligge, M., Solanki, S. K., \& Unruh, Y. C. 2000, A\&A, 353, 380

Floyd, L. 1999, Adv. Sp. Res., 23, 1459

Floyd, L. E., Herring, L. C., Prinz, D. K., \& Brueckner, G. E. 1996, in Ultraviolet Atmospheric and Space Remote Sensing: Methods and Instrumentation, Proc. SPIE, 2831, 36

Floyd, L. E., Herring, L. C., Prinz, D. K., \& Crane, P. C. 1998, in Optical Systems Contamination and Degradation, Proc. SPIE, 3427, 445

Floyd, L., Rottman, G., DeLand, M., \& Pap, J. 2003a, ESA SP-535, 195

Floyd, L. E., Cook, J. W., Herring, L. C., \& Crane, P. C. 2003b, Adv. Sp. Res., 31,2111

Floyd, L., Newmark, J., Cook, J., Herring, L., \& McMullin, D. 2005, J. Atm. Terr. Phys., 67, 3

Fontenla, J., White, O. R., Fox, P. A., Avrett, E. H., \& Kurucz, R. L. 1999, ApJ, 518,480

Fontenla, J. M., Harder, J., Rottman, G., et al. 2004, ApJ, 605, L85

Foster, S. 2004, Ph.D. Thesis, University of Southhampton, Faculty of Science, School of Physics and Astronomy

Fröhlich, C., Andersen, B., Appourchaux, T., et al. 1997, Sol. Phys., 170, 1

Haberreiter, M., Krivova, N. A., Schmutz, W., \& Wenzler, T. 2005, Adv. Sp. Res., 35, 365

Haigh, J. D. 1994, Nature, 370, 544

Haigh, J. D. 1996, Science, 272, 981

Haigh, J. D. 1999, J. Atm. Terr. Phys., 61, 63

Haigh, J. D. 2001, Science, 294, 2109

Heath, D. F., \& Schlesinger, B. M. 1986, JGR, 91, 8672

Kodera, K. 2004, GRL, 31, L24209

Kodera, K., \& Kuroda, Y. 2002, JGR (Atmospheres), 107 (D24), 4749

Krivova, N. A., \& Solanki, S. K. 2005, Mem. S. A. It., 76, 834

Krivova, N. A., Solanki, S. K., Fligge, M., \& Unruh, Y. C. 2003, A\&A, 399, L1

Larkin, A., Haigh, J. D., \& Djavidnia, S. 2000, Sp. Sci. Rev., 94, 199

Lean, J. 1989, Science, 244, 197

Lean, J. 2000, GRL, 27, 2425

Lean, J., Beer, J., \& Bradley, R. 1995, GRL, 22, 3195

Lean, J. L., Rottman, G. J., Kyle, H. L., et al. 1997, JGR, 102, 29939

Lean, J. L., Cook, J., Marquette, W., \& Johannesson, A. 1998, ApJ, 492, 390

Lockwood, M., \& Stamper, R. 1999, GRL, 26, 2461

Matthes, K., Langematz, U., Gray, L. L., Kodera, K., \& Labitzke, K. 2004, JGR (Atmospheres), 109 (D6), 6101

Mitchell, W. E., \& Livingston, W. C. 1991, ApJ, 372, 336

Prinz, D. K., Floyd, L. E., Herring, L. C., \& Brueckner, G. E. 1996, in Ultraviolet Atmospheric and Space Remote Sensing: Methods and Instrumentation, Proc. SPIE, 2831, 25

Rind, D. 2002, Science, 296, 673

Rottman, G. J. 1988, Adv. Sp. Res., 8, 53

Rottman, G. J., Woods, T. N., \& Sparn, T. P. 1993, JGR, 98 (D6), 10667

Rozanov, E. V., Schlesinger, M. E., Egorova, T. A., et al. 2004, JGR (Atmospheres), 109 (D1), 1110

Scherrer, P. H., Bogart, R. S., Bush, R. I., et al. 1995, Sol. Phys., 162, 129

Solanki, S. K., \& Unruh, Y. C. 1998, A\&A, 329, 747

Solanki, S. K., \& Fligge, M. 1999, GRL, 26, 2465

Solanki, S. K., \& Krivova, N. A. 2004, Sol. Phys., 224, 197

Solanki, S. K., Krivova, N. A., \& Wenzler, T. 2005, Adv. Sp. Res., 35, 376

Tobiska, W. K., Woods, T., Eparvier, F., et al. 2000, J. Atm. Terr. Phys., 62, 1233

Unruh, Y. C., Solanki, S. K., \& Fligge, M. 1999, A\&A, 345, 635

Unruh, Y. C., Solanki, S. K., \& Fligge, M. 2000, Sp. Sci. Rev., 94, 145

Viereck, R. A., \& Puga, L. C. 1999, JGR, 104, 9995

Viereck, R., Puga, L., McMullin, D., et al. 2001, GRL, 28, 1343

Viereck, R. A., Floyd, L. E., Crane, P. C., et al. 2004, Space Weather, 2

Wang, Y.-M., Lean, J. L., \& Sheeley, N. R. 2005, ApJ, 625, 522

Wenzler, T., Solanki, S. K., Krivova, N. A., \& Fluri, D. M. 2004, A\&A, 427, 1031

Wenzler, T., Solanki, S. K., \& Krivova, N. A. 2005a, A\&A, 432, 1057

Wenzler, T., Solanki, S. K., Krivova, N. A., \& Fröhlich, C. 2005b, A\&A, in preparation

Woods, T. 2002, ESA SP-508, 165

Woods, T. N., Prinz, D. K., Rottman, G. J., et al. 1996, JGR, 101 (D6), 9541

Woods, T. N., Eparvier, F. G., Bailey, S. M., et al. 2005, JGR (Space Physics), 110 (A9), 1312

Worden, J. R., White, O. R., \& Woods, T. N. 1998, ApJ, 496, 998

Worden, J. R., Woods, T. N., \& Bowman, K. W. 2001, ApJ, 560, 1020 